\title{
Dynamic OpenFlow-based Lightpath Restoration in Elastic Optical Networks on the GENI Testbed
}

\author{
(Invited Paper) \\ Lei Liu, Member, IEEE, Wei-Ren Peng, Ramon Casellas, Senior Member, IEEE, Takehiro Tsuritani, \\ Itsuro Morita, Member, IEEE, Ricardo Martínez, Raül Muñoz, Senior Member, IEEE, Masatoshi \\ Suzuki, Fellow, IEEE, Fellow, OSA, S. J. B. Yoo, Fellow, IEEE, Fellow, OSA
}

\begin{abstract}
Elastic optical networking (EON), with its flexible use of the optical spectrum, is a promising solution for future metro/core optical networking. For the deployment of EON in a real operational scenario, the dynamic lightpath restoration, driven by an intelligent control plane, is a necessary network function. Dynamic restoration can restore network services automatically, and thus greatly reduce the operational cost, compared with traditional manual or semi-static lightpath restoration strategies enabled by network operators via a network management system. To this end, in this paper, we present OpenFlow-enabled dynamic lightpath restoration in elastic optical networks, detailing the restoration framework and algorithm, the failure isolation mechanism, and the proposed OpenFlow protocol extensions. We quantitatively present the restoration performance via control plane experimental tests on the Global Environment for Network Innovations (GENI) testbed.
\end{abstract}

Index Terms - Software-Defined Networking (SDN), OpenFlow, Global Environment for Network Innovations (GENI), restoration, elastic optical network

Manuscript received October 6, 2014. This work was supported in part by BBN under the GENI 4 subcontract 1953 under NSF CNS-1346688, by DOE under the grant DE-FC02-13ER26154, by NSF under the EECS grant 1028729, and by DARPA DSO under the grants HR0011-11-1-0005 and W911NF-12-1-0311. The work of CTTC authors was partially supported by the European Community's Seventh Framework Programme FP7/2007-2013 through the IDEALIST project (grant agreement n. 317999), the EU-Japan coordinated project STRAUSS (grant agreement n. 608528), and the Spanish Ministry of Economy and Competitiveness (MINECO) through the project FARO (TEC2012-38119).

L. Liu and S. J. B. Yoo are with the University of California, Davis, One Shields Ave, Davis, CA 95616, USA (e-mail: liulei@ieee.org, sbyoo@ucdavis.edu).

W-R. Peng is with the Futurewei Technologies, 2330 Central Expressway, Santa Clara, CA 95050, USA (e-mail: Wei.Ren.Peng@huawei.com)

R. Casellas, R. Martínez and R. Muñoz are with the Centre Tecnològic de Telecomunicacions de Catalunya (CTTC), Castelldefels, 08860 Barcelona, Spain (e-mail: ramon.casellas@cttc.es, ricardo.martinez@cttc.es, raul.munoz@cttc.es).

T. Tsuritani, I. Morita and M. Suzuki are with the KDDI R\&D Laboratories Inc., 2-1-15 Ohara, Fujimino-shi, Saitama 356-8502, Japan (email: tsuri@,kddilabs.jp, morita@kddilabs.jp, suzuki@kddilabs.jp).

\section{INTRODUCTION}

$\mathrm{T}$ HE optical metro and core networks are evolving from the traditional fixed wavelength grid to a flexible grid paradigm [1]. Such networks are usually referred to as flexigrid optical networks or elastic optical networks (EONs) [2]. In EON, the optical spectrum of a lightpath (i.e., an optical connection) can be adaptively allocated in multiples of a basic spectrum slot width (e.g., $12.5 \mathrm{GHz}$ ) according to the traffic demands [3]. Compared with the traditional fixed-grid optical networking, such as the wavelength switched optical network (WSON), EON can greatly improve the network resource efficiency, reduce the network cost, and agilely handle traffic variations and large traffic (i.e. super-channels) [4, 5]. Therefore, the EON is a promising technology for future metro/core optical networks.

The role of a control plane is critically important in EONs, not only for connection provisioning, but also for restoring services, either in case of link failures or signal quality degradation. Recently, Software-Defined Networking (SDN) and, in particular, the OpenFlow (OF) [6] architecture and its associated protocol have attracted strong interest from both academia and industry, and many studies have extended OpenFlow to control optical networks [7-15]. The centralized SDN /OpenFlow-based control plane provides flexibility for the operators to control a network given its open interfaces and the open nature of its source code [16, 17].

Although a number of previous works have investigated OpenFlow-based EONs, these works mainly focused on dynamic connection provisioning [18-21]. To the best of our knowledge, only the works in $[22,23]$ have investigated OpenFlow-enabled restoration in EONs. Ref. [22] presented a proof-of-concept demonstration of OpenFlow-controlled dynamic recovery for only one failed connection on a real EON testbed employing a flexible transmitter (Tx) and receiver (Rx). Ref. [23] proposed a fast OpenFlow-based restoration scheme to minimize the parallel hardware configuration delay. However, physical impairments in the network are not considered in [23]. Since the restoration path usually has a different length and hop count compared to the 
working path, the consideration of physical layer impairments, modulation formats, or even bandwidth squeezed restoration [24] is important in order to attain successful service recovery.

In light of the above, in this work, we investigate OpenFlow-based dynamic lightpath restoration in EONs by considering all the above factors into account. We propose an OpenFlow-based failure isolation mechanism and a new OpenFlow OFPT_ALARM message. Based on these, a twophase restoration routing, spectrum, and modulation format assignment (RSMA) algorithm is presented. More importantly, control plane experimental tests are carried out on the Global Environment for Network Innovations (GENI) [25] testbed, which includes many GENI racks, regional and national backbone networks around the United States. This allows validating the overall feasibility of the approach and provides valuable insights into its potential for possible deployment in the future.

The rest of this paper is organized as follows: Section II presents the network architecture of OpenFlow-controlled EON with flexible transmitters and receivers. Section III introduces the key enabling techniques. Section IV reports the testbed setup and results. We conclude in Section $\mathrm{V}$ by summarizing our contributions.

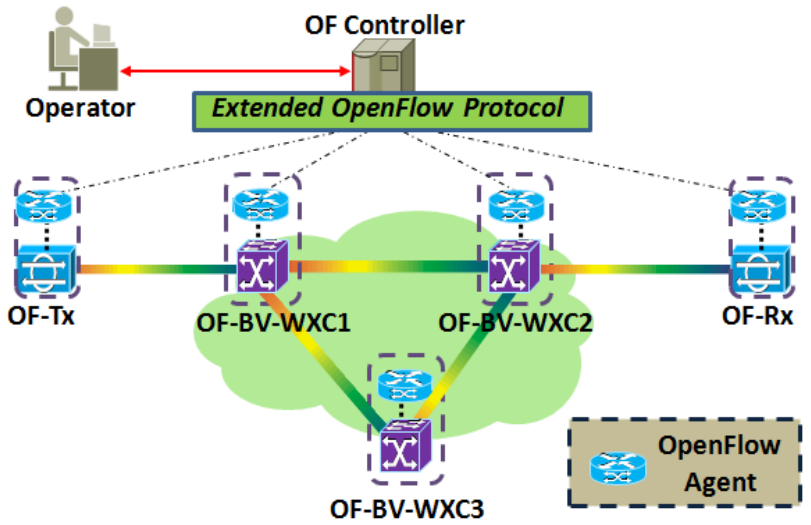

Fig. 1. Network architecture for OpenFlow-controlled EON.

\section{NETWORK ARCHITECTURE}

The data plane of an OpenFlow-based EON includes bandwidth-variable wavelength cross-connects (BV-WXCs) and flexible transmitters/receivers (Txs/Rxs), as shown in Fig.1. As illustrated in [3], the BV-WXC is implemented by using multiple bandwidth-variable wavelength selective switch (BV-WSS). The flexible Txs/Rxs are able to send/receive optical signals with different bit rates and modulation formats without hardware modification. As mentioned in our previous work [20, 26], the Tx/Rx utilize the direct-detected optical orthogonal frequency division multiplexing (DDO-OFDM) $[27,28]$ technique. However, it is important to note that the proposed restoration scheme applies to any other transmission technique such as coherent OFDM, coherent optical wavelength-division multiplexing (WDM) or Nyquist WDM.

Fig.2(a) and Fig.2(b) describe the Tx and Rx processing for DDO-OFDM supported by advanced digital signal processing respectively. At the Tx, the random binary bits are firstly mapped to the pre-defined Quadrature Amplitude Modulation (QAM) symbols such as 4-, 16-, or 64QAM, and, along with a radio frequency $(\mathrm{RF})$ pilot tone [29], converted to time domain using a 128-point Inverse Fast Fourier Transform (IFFT). A cyclic prefix with a length of $\sim(1 / 16 \times$ symbol duration $)$ is added at the head of each OFDM symbol to prevent the intersymbol interference (ISI). After that, $4 \mathrm{x}$ oversampling is performed to model the output analog signals to increase the reliability of the results. The generated signal is used to drive a linear IQ modulator fed by a narrow-linewidth laser. The output of the modulator is sent to the EON. At the Rx, a square-law photodiode is used to down-convert the received signals to the electrical baseband. After down-sampling (by analog-to-digital converter, ADC), signal processing such as cyclic prefix removal, FFT, channel estimation and equalization, QAM de-mapping are performed to recover the transmitted signals.

As Fig. 1 Illustrates, an OpenFlow agent which implements the extended OpenFlow protocol will be on top of each network element (NE), including NEs with a BV-WXC, Tx and Rx. The combination of the OpenFlow agent and its corresponding data plane hardware is referred to as OpenFlow-enabled BV-WXC (OF-BV-WXC), OpenFlowenabled transmitter (OF-Tx) and OpenFlow-enabled receiver (OF-Rx) respectively. A centralized OF controller will communicate with all OpenFlow agents through the extended OpenFlow protocol, and in turn, control the hardware in the data plane.
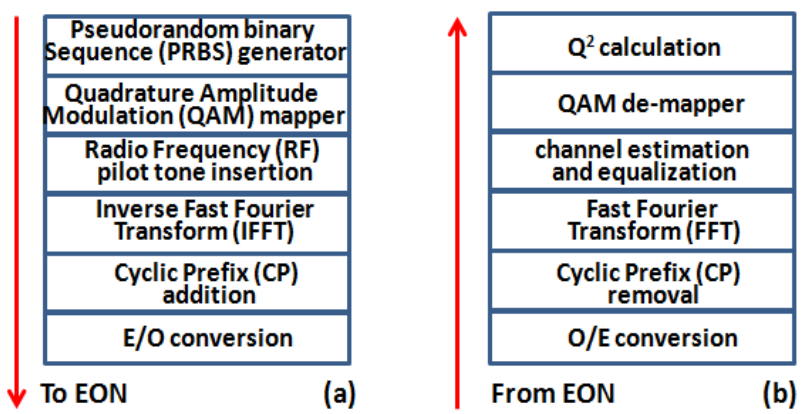

Fig. 2. (a) Tx and (b) Rx processing for DDO-OFDM supported by advanced digital signal processing.

\section{Key ENABLING TECHNIQUES}

\section{A. OpenFlow Protocol Extensions}

The OpenFlow protocol is originally designed for packetswitched networks. Therefore, in order to control an EON, the OpenFlow protocol needs to be extended with EON features. The key extensions, as investigated in previous works [18], include:

(1) The OFPT_FEATURES_REPLY message is extended to advertise the features of an EON node (e.g., flexi-grid switching capability, neighbor information, available spectrum slots, etc.) to the OF controller;

(2) The OFPT_PACKET_IN message is extended to carry the bit rate information of the incoming client traffic, and used 
to trigger the establishment of the optical connection;

(3) The OFPT_FLOW_MOD message is extended to encapsulate the RSMA results from the OF controller, including input/output ports, central frequency, assigned spectrum slots, and modulation format, etc., for each OpenFlow agent to control the underlying hardware.

In addition to the above protocol extensions, we also need to introduce a new OpenFlow protocol extension to notify link failures, enabling dynamic restoration. This can be achieved by either extending the OFPT_PORT_STATUS message or introducing a new OFPT_ALARM message. In this work, we used the latter approach and Fig.3 illustrates the packet format of such a new OFPT_ALARM message. Similarly with the concept of "unnumbered link" from GMPLS, the OFPT_ALARM message carries the Datapath ID field (8 bytes) for indicating the node address, and an interface ID field ( 4 bytes) for indicating the port associated to the failed link. Once the OF controller receives an OFPT_ALARM message, it can identify a potentially failed link from the Datapath ID and interface ID information encapsulated in this message. However, it should be noted that for a given connection, an upstream link failure may cause all downstream BV-WXCs to send out alarms. Therefore, a failure isolation mechanism is required by the OF controller to determine the exact failed point, as will be detailed in section III.B.

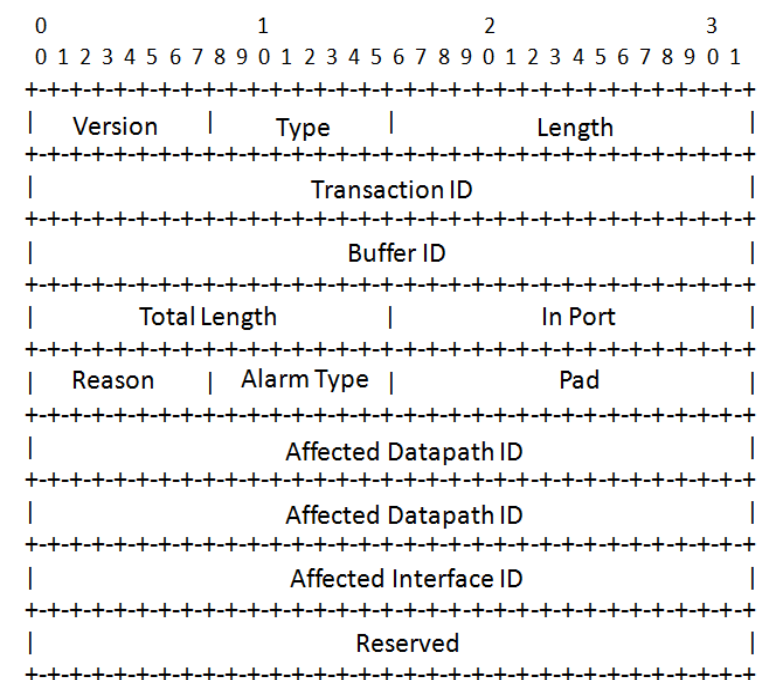

Fig. 3. The proposed OFPT_ALARM message.

\section{B. Failure Isolation Mechanism}

We assume that each BV-WXC is equipped with optical power monitors to detect the loss of light when a link failure happens. In this case, an upstream link failure may cause all downstream BV-WXCs to send out alarms for a given connection. As shown in the example in Fig.4, once a link failure occurs between the link WXC2-3, affecting an existing connection Tx-WXC1-2-3-4-Rx, both WXC3 and WXC4 will send out OFPT_ALARM messages to the OF controller, reporting the loss of light failure on links WXC2-3 and
WXC3-4 respectively. Therefore, the OF controller needs to run a failure isolation mechanism to determine the exact failed point. Thanks to the centralized architecture, the OF controller can easily understand the entire network information for its global view. Although a control node in a distributed control plane architecture such as GMPLS can also have a global view through dynamic dissemination of Open Shortest Path FirstTraffic Engineering (OSPF-TE) link state advertisement (LSA), such an operation is more complicated and accompanies higher overhead. With this global view, the OF controller can combine these affected links into a segment to check if it belongs to existing connections. If true, the OF controller localizes the first link of the segment as the failed point. As the example in Fig.4 illustrates, the OF controller receives OFPT_ALARM messages to report the loss of light on links WXC2-3 and WXC3-4 respectively, and then the OF controller can combine the affected links into a segment WXC2-3-4. After that, the OF controller proceeds to check if the segment WXC2-3-4 belongs to any lightpath connections or not. In this example, it belongs to an existing connection Tx-WXC1-2-3-4-Rx. Therefore, the OF controller can localize the first link of the segment, i.e., the link WXC2-3, as the exact failure point.

Note that, the control plane is commonly using the carriers' data communications network (DCN), which has a transmission delay for control messages, usually ranging from several milliseconds to several tens of milliseconds depending on the network size and the hop count. In this case, the OFPT_ALARM messages, generated by different nodes for a given link failure event, may arrive at the OF controller at different times. As the proposed failure isolation mechanism has to collect all the OFPT_ALARM messages to make an exact decision on the failed point, waiting time $\boldsymbol{\Delta t}$ is required by the OF controller to receive all the corresponding alarm messages for a link failure event. That is to say, once an OFPT_ALARM message is received, the OF controller will wait for a time period $\boldsymbol{\Delta t}$ to collect other alarm messages, and make a decision after $\boldsymbol{\Delta t}$ expires. The selection of $\boldsymbol{\Delta} \boldsymbol{t}$ value can be made by the network carriers according to the network size and the placement of the OF controller. In addition, the processing latency of controller and the possible message queue delay may also affect the time for failure isolation. Considering these factors and assuming that the control message propagation times from $\mathrm{WXC1}, 2, \ldots \mathrm{N}$ to the $\mathrm{OF}$ controller are $t_{1}, t_{2}, \ldots t_{N}$ respectively, $\Delta t$ can be selected slightly larger than the maximum value of $t_{1}, t_{2}, \ldots t_{N}$, which is the worst case that the OF controller has to wait to receive all the failure alarms for a link failure event. 


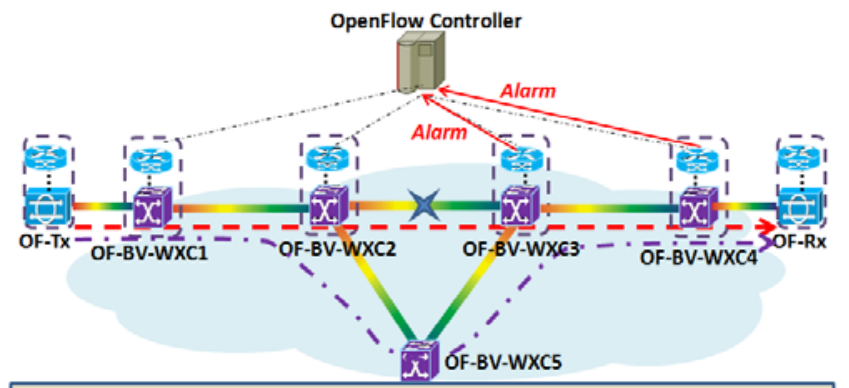

Link failure $---->$ Working path $-\cdots-\cdots$ Restoration path

Fig. 4. OpenFlow-based lightpath restoration.

\section{Dynamic Restoration Procedure}

When the physical link failure happens, all the flow entries in the flow tables for the affected connections still exist. Therefore, from the control plane point of view, after the link failure happens, the OF controller needs to decide (a) to delete the flow entries for affected connections firstly or (b) to provision the new connection firstly. The case (a) is usually referred to break-before-make (BBM) and the case (b) is referred to as make-before-break (MBB). The proposed method adopts the BBM scheme, wherein the working path is deleted prior to set up the restoration path. Compared with the MBB scheme, the BBM can cause a longer latency for restoration since the working path has to be released firstly, but it is easier to manage the flow entries in the BBM scheme, and the released resources can be used immediately by other incoming connections, which is also helpful for effective network resource utilization. Once an OFPT_ALARM message is received, the OF controller starts to run the failure isolation mechanism detailed in section III.B, and restore the affected connections, releasing them and setting them up with a new path that excludes the failed link. For this, after the failure point is identified, the OF controller performs a twophase restoration RSMA algorithm (as detailed in section III.D), and then controls corresponding Tx, Rx and WXCs to create a restoration path through extended OFPT_FLOW_MOD messages.

\section{Two-Phase Restoration RSMA Algorithm}

Considering the fact that fully dynamic RSMA computation is complex and is likely to be CPU-intensive, as well as that a single link failure may affect multiple connections, to reduce restoration time and to mitigate the effect of centralized restoration, we implement a two-phase restoration RSMA algorithm in the OF controller, and we use the $Q^{2}$ factor [27] as the signal quality indicator.

In the first phase, executed off-line, the $K$-shortest paths for each source-destination node pair are calculated. After that, pre-computed tables comprise the $Q^{2}$ factor values (in $\mathrm{dB}$ ) and optical spectrum width (in $\mathrm{GHz}$ ) information for given net data rates, modulation formats, path distances and hop counts are generated, which are the results of the simulation of DDOOFDM transmission. For longer paths not considered during the off-line planning, the most robust modulation format is assigned to guarantee the signal quality at the $\mathrm{Rx}$. The second phase, during the actual dynamic restoration, encompasses path selection and resource assignment when a failure alarm is received and the exact failure point is identified. The OF controller performs a lookup for pre-computed static planning tables and checks the pre-computed paths which do not include the failed link as the potential restoration paths. In this step, the OF controller firstly tries to find restoration paths that can support the same data rate of the working path. If such a restoration path does not exist, the OF controller then reduces the data rate and checks static planning tables again (i.e. bandwidth squeezed restoration). The OF controller serves failed connections one by one, and if multiple combinations are possible with the same data rate, the criterion is to minimize the resulting optical spectrum.

\section{EXPERIMENTAL SETUP, Results AND Discussion}

We set up an OpenFlow control plane network over the GENI testbed by using GENI racks [25], as shown in Fig.5, which is a snapshot of the FLACK GUI [25]. Note that it is a testbed with only OpenFlow-based control plane without any real associated optical hardware in the data plane. The data plane topology is emulated by using the control plane. As shown in Fig.5, the emulated data plane comprises 14 BVWXC nodes, which are connected through emulated fiber links with different lengths. All the nodes are connected to a dedicated OF controller based on NOX [30] running in Linux Ubuntu 10.04. The DWDM links are characterized by 128 individual slots of $6.25 \mathrm{GHz}$ each. Linear standard single mode fiber (SSMF) links are assumed, featuring an attenuation of $0.2 \mathrm{~dB} / \mathrm{km}$ and a chromatic dispersion coefficient of 16 $\mathrm{ps} / \mathrm{nm} \bullet \mathrm{km}$. The input power to fibers is $0 \mathrm{dBm}$. We use $K=3$ for the $K$-shortest paths algorithm, restricted to a maximum number of 5 hops. For each link, the maximum span length is limited to $80 \mathrm{~km}$. Each span contains an erbium doped fiber amplifier (EDFA) compensating exactly the loss of each span with a fixed noise figure of $6 \mathrm{~dB}$. Each ROADM is modeled as a 4th-order Gaussian-shaped filter with its 3dB-bandwidth covering $\sim 1.5$ times the signal bandwidth. The target $Q^{2}$ factor is $8.53 \mathrm{~dB}$ [27] which is the threshold of the hard-decision forward error correction. According to our experimental setup in Fig. 5, we measure the maximum latency from a node to the OF controller, which is slightly less than $5 \mathrm{~ms}$. Therefore, according to the policy that we presented in section III.B, in our experiment, we set $\Delta t$ as $5 \mathrm{~ms}$. Note that the selection of $\Delta t$ may affect the performance. If $\Delta t$ is too small, the OF controller may not be able to collect all the failure alarms, which may lead to incorrect failure isolation and thus reduce the successful rate of the restoration. If $\Delta t$ is too large, the restoration latency is increased accordingly. Different modulation formats such as 4-, 16- and 64-QAM, and various data rates including 10,40 , and $100 \mathrm{~Gb} / \mathrm{s}$ are discussed. 


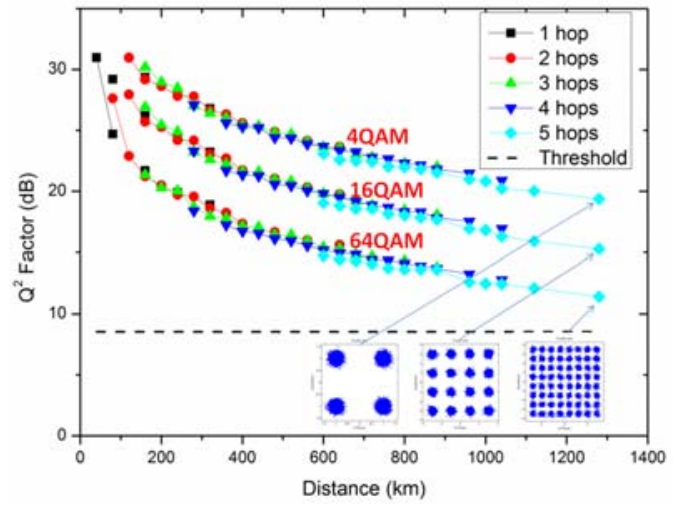

Fig. 6. $\mathrm{Q}^{2}$ factor (in $\mathrm{dB}$ ) at the $\mathrm{Rx}$ for different distances and hop count corresponding to different modulation formats (4QAM, 16QAM, 64QAM) at $10 \mathrm{~Gb} / \mathrm{s}$ DDO-OFDM transmission.

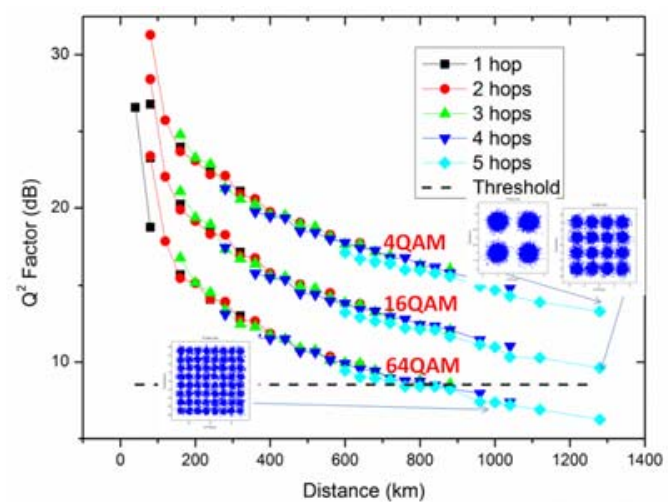

Fig. 7. $\mathrm{Q}^{2}$ factor (in $\mathrm{dB}$ ) at the $\mathrm{Rx}$ for different distances and hop count corresponding to different modulation formats (4QAM, 16QAM, 64QAM) at 40Gb/s DDO-OFDM transmission.

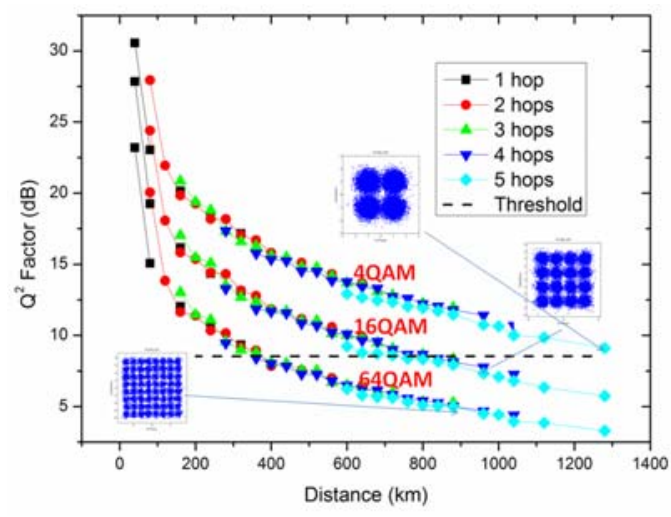

Fig. 8. $Q^{2}$ factor (in $\mathrm{dB}$ ) at the $\mathrm{Rx}$ for different distances and hop count corresponding to different modulation formats (4QAM, 16QAM, 64QAM) at
$100 \mathrm{~Gb} / \mathrm{s}$ DDO-OFDM transmission.

Fig.6, Fig. 7 and Fig.8 show the off-line network planning results for the restoration paths, which are obtained via Matlab simulation. $Q^{2}$ factors at the Rx side are measured for different distances and hop counts corresponding to different modulation formats (4-, 16-, 64-QAM) at 10, 40, and $100 \mathrm{~Gb} / \mathrm{s}$ DDO-OFDM transmissions. Table 1 shows the signal bandwidth for DDO-OFDM with different modulation formats. As expected, the 4QAM constellation is more robust to physical impairments, whereas the 64QAM constellation gives the highest spectral efficiency. It can be observed that all the 4QAM cases achieve above the targeted $Q^{2}$ factor threshold, whereas when using 16QAM at $100 \mathrm{~Gb} / \mathrm{s}, 64 \mathrm{QAM}$ at $40 \mathrm{~Gb} / \mathrm{s}$, and 64QAM at $100 \mathrm{~Gb} / \mathrm{s}$, the attainable distance is limited. These results are stored in the pre-computed static planning tables in the NOX for dynamic restoration path selection. Once the off-line network planning tables are produced, in this work, there is no further verification of any transmission properties during the dynamic restoration procedure, due to the lack of optical gears (e.g., optical performance monitors) in the GENI testbed.

TABLE I

SIGNAL BANDWIDTH AND REQUIRED SPECTRUM SLOTS FOR DDO-OFDM WITH DIFFERENT MODULATION FORMATS

\begin{tabular}{|c|c|c|c|c|}
\hline $\begin{array}{l}\text { Modulation } \\
\text { Format }\end{array}$ & & $10 \mathrm{G} \mathrm{bit} / \mathrm{s}$ & $40 \mathrm{G} \mathrm{bit/s}$ & $100 \mathrm{G} \mathrm{bit} / \mathrm{s}$ \\
\hline \multirow{2}{*}{ 4QAM } & $\begin{array}{c}\text { Signal } \\
\text { Bandwidth } \\
(\mathrm{GHz})\end{array}$ & 10.625 & 42.5 & 106.25 \\
\hline & $\begin{array}{c}\text { Number of } \\
\text { Spectrum } \\
\text { Slots }^{\mathrm{a}}\end{array}$ & 2 & 7 & 17 \\
\hline \multirow{2}{*}{ 16QAM } & $\begin{array}{c}\text { Signal } \\
\text { Bandwidth } \\
(\mathrm{GHz})\end{array}$ & 5.3125 & 21.25 & 53.125 \\
\hline & $\begin{array}{c}\text { Number of } \\
\text { Spectrum } \\
\text { Slots }^{\mathrm{a}}\end{array}$ & 1 & 4 & 9 \\
\hline \multirow{2}{*}{ 64QAM } & $\begin{array}{c}\text { Signal } \\
\text { Bandwidth } \\
(\mathrm{GHz})\end{array}$ & 3.5417 & 14.1667 & 35.4167 \\
\hline & $\begin{array}{c}\text { Number of } \\
\text { Spectrum } \\
\text { Slots }^{\mathrm{a}}\end{array}$ & 1 & 3 & 6 \\
\hline
\end{tabular}

${ }^{\mathrm{a}}$ Each spectrum slot: $6.25 \mathrm{GHz}$ 


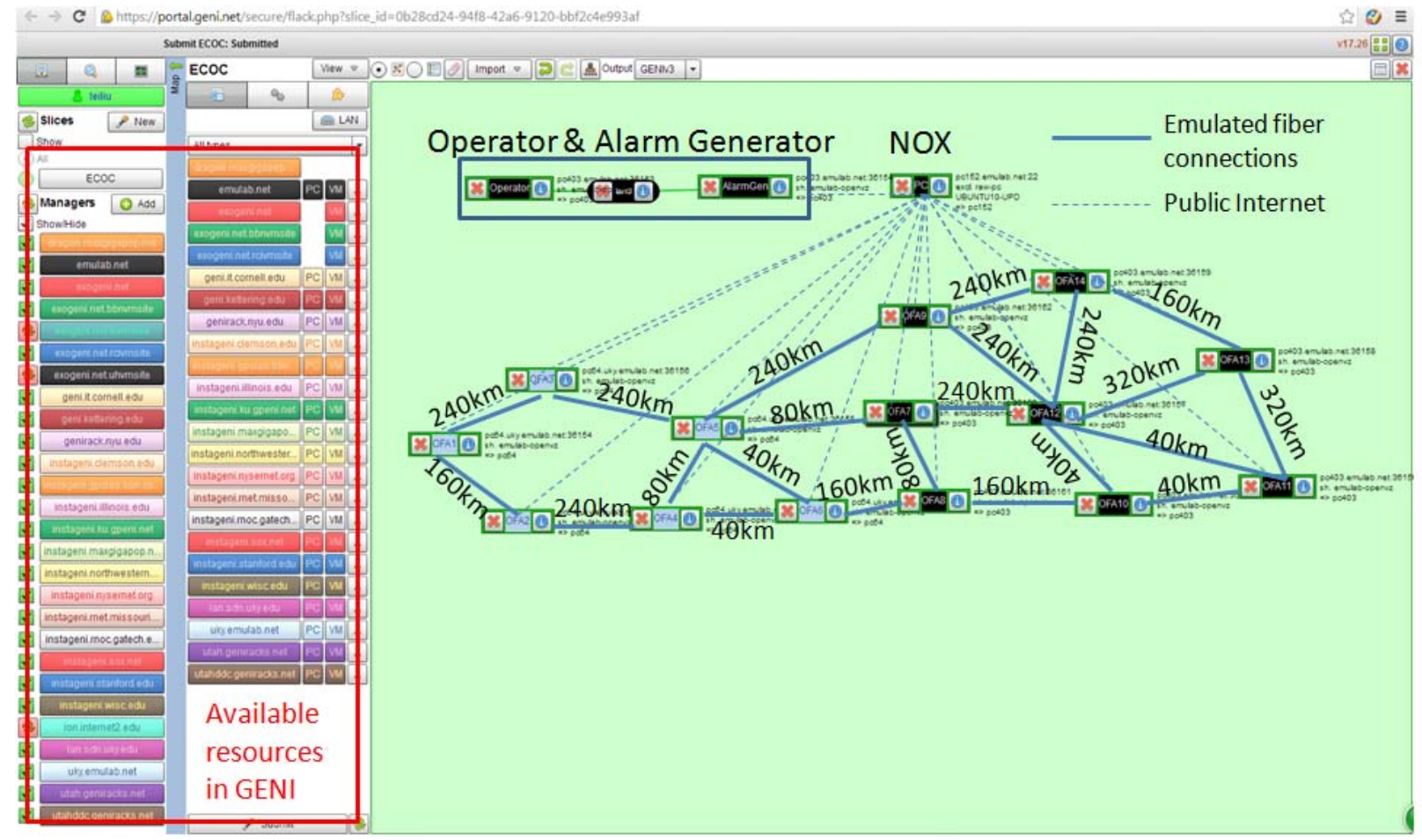

Fig. 5. Experimental setup on GENI testbed.

After the off-line network planning results for the restoration paths are obtained, we conduct control plane experimental tests to measure the performance of the proposed restoration approach over the GENI testbed. Compared with a lab environment, the experimental tests on the GENI testbed allow the control plane performance to be obtained over a real national backbone network, which can facilitate to validate the overall feasibility of the approach and provide valuable insights into its potential for possible deployment in a real operational scenario in future. In the control plane experimental tests, we firstly configure the connection database in the NOX controller to make sure the average link resource utilization is $30 \%, 40 \%, 50 \%$ and $60 \%$ respectively, and then the NOX sets up these connections according to the connection database. The placement of connections is uniformly distributed among all the candidate links. Given the $30 \%$ case as an example, if the whole network has 5,632 spectrum slots in total, then we set up a number of connections so that $\sim 1,690$ spectrum slots are occupied by connections. The source, destination, modulation format, and bit rate of these connections are all randomly selected from the candidate pool. After these connections are established, we randomly select a link as the failed point and directly send the OFPT_ALARM messages to the NOX to emulate link failure events. This failed link is randomly and uniformly distributed among all the links. Fig.9 shows the Wireshark capture of an OFPT_ALARM message, which encapsulates the alarm type and the identifier of the failed link. When such a failure alarm is received, the NOX performs the two-phase RSMA computation as detailed above. If a restoration path is found, the NOX controls the nodes to set up the restoration path through extended OFPT_FLOW_MOD messages. We introduce one failure each time and count the number of affected connections, and then we repeat this procedure from scratch until $\sim 1000$ connections have been affected in different link utilization scenarios in total. We measure the control plane processing latency and the restorability for dynamic restoration on the given network scenario, as shown in Fig.10 and Fig. 11 respectively. Note that these results are measured on an OpenFlow control plane testbed, and optical hardware gears for elastic optical networking are not included. Therefore, the restoration latencies do not take into account hardware configuration delay. As previously investigated in $[18,31]$, the control and configuration of elastic optical network data plane hardware may need several tens to hundreds of milliseconds, which should be added to the overall restoration latency if the data plane hardware is included. For comparison purposes we also measure the performance in Fig.11 with the same experimental setup mentioned above, of the previously proposed OpenFlowenabled dynamic restoration approach, which does not consider the physical impairments and bandwidth squeezed restoration (such as [23]). These performance results validate the overall feasibility of the proposed OpenFlow-controlled restoration in an EON testbed emulated by the GENI infrastructure, and the proposed dynamic restoration approach outperforms the previous OpenFlow-based restoration in terms of restorability. One of the reasons is that, by using the 
previous OpenFlow-based restoration approach, even if the OF controller successfully finds a restoration path, this path may not have a satisfied $Q^{2}$ factor at the receiver side, which results in a lower restorability.

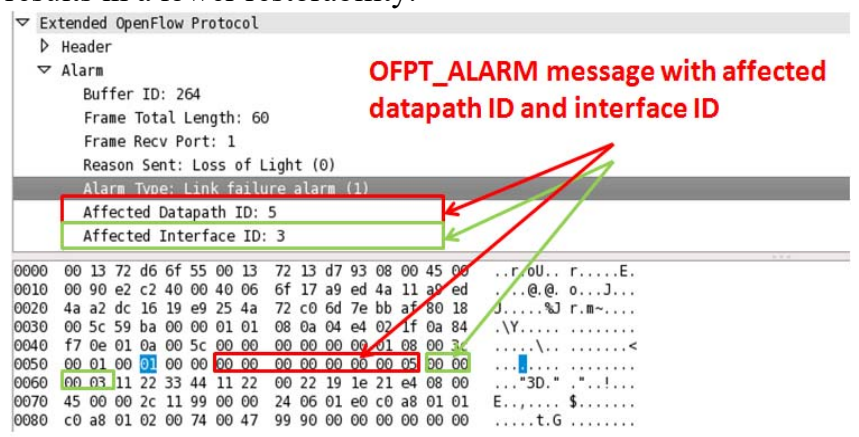

Fig. 9. Wireshark capture of the new OFPT_ALARM message.

Macroscopically, from a control plane perspective, the centralized SDN/OpenFlow controller has some similar features (e.g. network topology or connection management) with the path computation element (PCE), in particular, the active stateful PCE [32], in which the PCE is able to affect (modify or suggest the modification of) the state of network connections. Both the OpenFlow controller and the active stateful PCE also complement each other, since a PCE is responsible mainly for path computation accessible via an open, standard, and flexible protocol (i.e. PCE communication protocol, PCEP [33]), and the OpenFlow controller performs the task of the actual data plane forwarding provisioning. In light of this, in our previous paper [34], we have proposed the design and implementation of a centralized control plane based on a stateful PCE, acting as an OpenFlow controller, to control optical networks. The functions proposed in this paper can also by applied by such a stateful PCE / OpenFlow integrated controller.

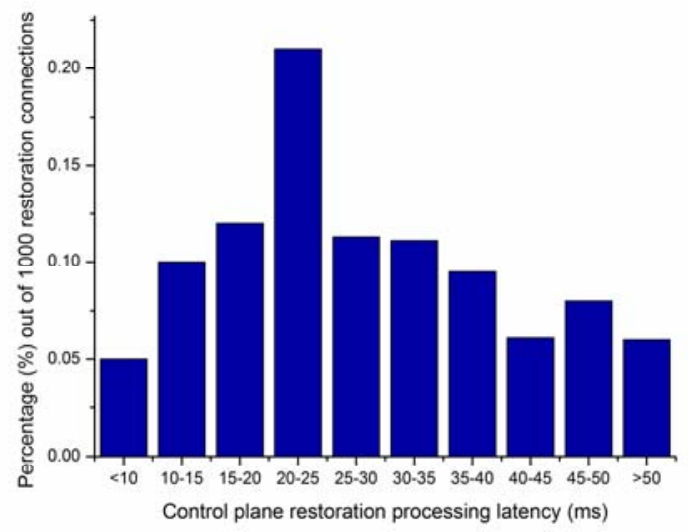

Fig. 10. Control plane restoration latency.

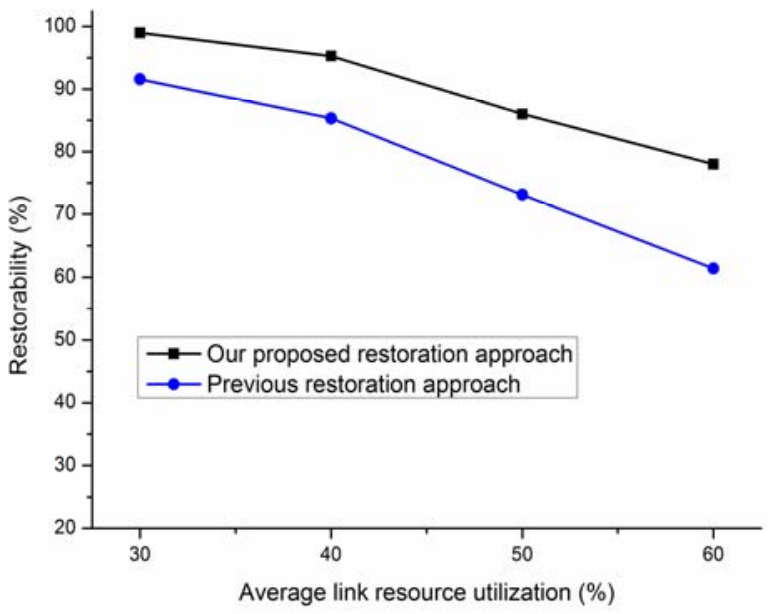

Fig. 11. Restorability under different network status (different resource utilization ratio).

\section{CONCLUSIONS}

In this paper, we present OpenFlow-based dynamic connection restoration for a software-defined EON. The overall feasibility and efficiency of the proposed solutions, including the control framework, the failure isolation mechanism, the restoration algorithm, and protocol extensions are validated and quantitatively evaluated in terms of restoration latencies and restorability on the GENI testbed.

In a more general context, the proposed restoration approach can be applied to IP over EON paradigm, where the OF controller can provide the unified control plane functions for both IP and the EON layers. The failure of optical components in the EON layer may lead to multiple link failures in the IP layer. In this case, the OF controller can also insert new flow entries into the corresponding IP routers to restore the IP flows, thanks to the global view of the controller. In addition, we also believe the proposed approach can coordinate and support the IP network which has the feature like fast reroute (FRR), but in this case, further studies regarding the cross-layer planning and algorithm design are needed [35], which will be our future works.

\section{REFERENCES}

ITU-T G.694.1, "Spectral grids for WDM applications: DWDM frequency grid," Feb. 2012.

O. Gerstel, M. Jinno, A. Lord, and S. J. B. Yoo, "Elastic optical networking: a new dawn for the optical layer?," IEEE Commun. Mag. , vol. 50, no. 2, pp. s12-s20, Feb. 2012.

M. Jinno, H. Takara, B. Kozicki, Y. Tsukishima, Y. Sone, and S. Matsuoka, "Spectrum-efficient and scalable elastic optical path network: architecture, benefits, and enabling technologies," IEEE Commun. Mag. , vol. 47, no. 11, pp. 66-73, Nov. 2009.

M. Klinkowski and K. Walkowiak, "On the advantages of elastic optical networks for provisioning of cloud computing traffic," IEEE Network, vol. 27, no. 6, pp. 44-51, Nov.-Dec. 2013.

M. Jinno, H. Takara, and Y. Sone, "Elastic optical path networking: enhancing network capacity and disaster survivability toward 1 Tbps era," in 16th Opto-Electronics And Communications Conference (OECC 2011), pp. 401-404, Kaohsiung, Taiwan, Jul. 2011.

OpenFlow. Available: http://www.openflow.org/ 
[7] L. Liu, T. Tsuritani, I. Morita, H. Guo, and J. Wu, "OpenFlowbased wavelength path control in transparent optical networks: a proof-of-concept demonstration," in European Conference and Exhibition on Optical Communication (ECOC 2011), paper Tu.5.K.2, Geneva, Switzerland, Sep. 2011.

[8] L. Liu, D. Zhang, T. Tsuritani, R. Vilalta, R. Casellas, L. Hong, I. Morita, H. Guo, J. Wu, R. Martinez, and R. Muñoz, "First field trial of an OpenFlow-based unified control plane for multi-layer multi-granularity optical networks," in Optical Fiber Communication Conference and Exposition and National Fiber Optic Engineers Conference (OFC/NFOEC 2012), paper PDP5D.2, Los Angeles, CA, USA, Mar. 2012.

[9] M. Channegowda, P. Kostecki, N. Efstathiou, S. Azodolmolky, R. Nejabati, P. Kaczmarek, A. Autenrieth, J.-P. Elbers, and D. Simeonidou, "Experimental evaluation of extended OpenFlow deployment for high-performance optical networks," in European Conference and Exhibition on Optical Communication (ECOC 2012), paper Tu.1.D.2, Amsterdam, Netherlands, Sep. 2012.

[10] J. Zhang, Y. Zhao, H. Yang, Y. Ji, H. Li, Y. Lin, G. Li, J. Han, Y. Lee, and T. Ma, "First demonstration of enhanced software defined networking (eSDN) over elastic grid (eGrid) optical networks for data center service migration," in Optical Fiber Communication Conference and Exposition and National Fiber Optic Engineers Conference (OFC/NFOEC 2013), paper PDP5B.1, Anaheim, CA, USA, Mar. 2013.

[11] F. Paolucci, F. Cugini, N. Hussain, F. Fresi, and L. Poti, "OpenFlow-based flexible optical networks with enhanced monitoring functionalities," in European Conference and Exhibition on Optical Communication (ECOC 2012), paper Tu.1.D.5, Amsterdam, Netherlands, Sep. 2012.

[12] R. Casellas, R. Martínez, R. Munoz, L. Liu, T. Tsuritani, and I. Morita, "An integrated stateful PCE / OpenFlow controller for the control and management of flexi-grid optical networks," in Optical Fiber Communication Conference and Exposition and National Fiber Optic Engineers Conference (OFC/NFOEC 2013), paper OW4G.2, Anaheim, CA, USA, Mar. 2013.

[13] S. Azodolmolky, R. Nejabati, E. Escalona, R. Jayakumar, N. Efstathiou, and D. Simeonidou, "Integrated OpenFlow - GMPLS control plane: an overlay model for software defined packet over optical networks " Opt. Express, vol. 19, no. 26, pp. B421-B428, Dec. 2011

[14] N. Cvijetic, A. Tanaka, P. N. Ji, S. Murakami, K. Sethuraman, and T. Wang, "First OpenFlow-based software-defined $\lambda$-flow architecture for flex-grid OFDMA mobile backhaul over passive optical networks with filterless direct detection ONUs," in Optical Fiber Communication Conference and Exposition and National Fiber Optic Engineers Conference (OFC/NFOEC 2013), paper PDP5B.2, Anaheim, CA, USA, Mar. 2013.

[15] L. Liu, D. Zhang, T. Tsuritani, R. Vilalta, R. Casellas, L. Hong, I. Morita, H. Guo, J. Wu, R. Martínez, and R. Muñoz, "Field trial of an OpenFlow-based unified control plane for multi-layer multigranularity optical switching networks," J. Lightw. Technol,, vol. 31, no. 4, pp. 506-514, Feb. 2013.

[16] L. Liu, T. Tsuritani, I. Morita, R. Casellas, R. Martínez, and R. Muñoz, "Control plane techniques for elastic optical networks: GMPLS/PCE vs OpenFlow," in IEEE Global Communications Conference (GLOBECOM 2012), pp. 352-357, Anaheim, CA, USA, Dec. 2012

[17] S. Das, G. Parulkar, and N. McKeown, "Why OpenFlow/SDN can succeed where GMPLS failed," in European Conference and Exhibition on Optical Communication (ECOC 2012), paper Tu.1.D.1, Amsterdam, Netherlands, Sep. 2012.

[18] L. Liu, R. Muñoz, R. Casellas, T. Tsuritani, R. Martínez, and I. Morita, "OpenSlice: an OpenFlow-based control plane fo spectrum sliced elastic optical path networks," Opt. Express, vol. 21, no. 4, pp. 4194-4204, September 16-20, 2012 Feb. 2012.

[19] M. Channegowda, R. Nejabati, M. R. Fard, S. Peng, N. Amaya, G. Zervas, D. Simeonidou, R. Vilalta, R. Casellas, R. Martínez, R. Muñoz, L. Liu, T. Tsuritani, I. Morita, A. Autenrieth, J.-P. Elbers, P. Kostecki, and P. Kaczmarek, "First demonstration of an OpenFlow based software-defined optical network employing packet, fixed and flexible DWDM grid technologies on an international multi-domain testbed," in European Conference and Exhibition on Optical Communication (ECOC 2012), paper PDP Th.3.D.2, Amsterdam, Netherlands, Sep. 2012.
[20] L. Liu, W.-R. Peng, R. Casellas, T. Tsuritani, I. Morita, R. Martínez, R. Muñoz, and S. J. B. Yoo, "OpenFlow-controlled elastic optical networks with direct-detection optical OFDM (DDO-OFDM) transmission," in European Conference and Exhibition on Optical Communication (ECOC 2013), paper We.1.E.4, London, UK, Sep. 2013.

[21] N. Sambo, G. Meloni, F. Paolucci, F. Cugini, M. Secondini, F. Fresi, L. Poti, and P. Castoldi, "Programmable transponder, code and differentiated filter configuration in elastic optical networks," J. Lightw. Technol., vol. 32, no. 11, pp. 2079-2086, Jun. 2014.

[22] L. Liu, H. Y. Choi, T. Tsuritani, I. Morita, R. Casellas, R. Martínez, and R. Muñoz, "First proof-of-concept demonstration of OpenFlow-controlled elastic optical networks employing flexible transmitter/receiver," in International Conference on Photonics in Switching (PS 2012), paper PDP1, Corsica, France, Sep. 2012.

[23] A. Giorgetti, F. Paolucci, F. Cugini, and P. Castoldi, "Fast restoration in SDN-based flexible optical networks," in Optical Fiber Communication Conference and Exposition (OFC), paper Th3B.2, San Francisco, CA, USA, Mar. 2014.

[24] Y. Sone, A. Watanabe, W. Imajuku, Y. Tsukishima, B. Kozicki, H. Takara, and M. Jinno, "Bandwidth squeezed restoration in spectrum-sliced elastic optical path networks (SLICE)," J. Opt Commun. Netw., vol. 3, no. 3, pp. 223-233 Mar. 2011.

[25] GENI. Available: https://www.geni.net/

[26] L. Liu, W.-R. Peng, R. Casellas, T. Tsuritani, I. Morita, R. Martínez, R. Muñoz, and S. J. B. Yoo, "Design and performance evaluation of an OpenFlow-based control plane for softwaredefined elastic optical networks with direct-detection optical OFDM (DDO-OFDM) transmission," Opt. Express, vol. 22, no. 1, pp. 30-40, Jan. 2014.

[27] W.-R. Peng, I. Morita, H. Takahashi, and T. Tsuritani, "Transmission of high-speed $(>100 \mathrm{~Gb} / \mathrm{s})$ direct-detection optical OFDM superchannel," J. Lightw. Technol., vol. 30, no. 12, pp. 2025-2034, Jun. 2012.

[28] W. Shieh, "OFDM for flexible high-speed optical networks," $J$. Lightw. Technol., vol. 29, no. 10, pp. 1560-1577, May 2011 Mar. 2011.

[29] W.-R. Peng, X. Wu, V. R. Arbab, K.-M. Feng, B. Shamee, L. C. Christen, J.-Y. Yang, A. E. Willner, and S. Chi, "Theoretical and experimental investigations of direct-detected RF-tone-assisted optical OFDM systems," J. Lightw. Technol., vol. 27, no. 10, pp. 1332-1339, May 2009.

[30] NOX. Available: http://www.noxrepo.org/

[31] F. Cugini, G. Meloni, F. Paolucci, N. Sambo, M. Secondini, L. Gerardi, L. Potì, and P. Castoldi, "Demonstration of flexible optical network based on path computation element," IEEE/OSA J. Lightw. Technol., vol. 30, no. 5, pp. 727-733, Mar. 2012.

[32] E. Crabbe, I. Minei, J. Medved, and R. Varga, "PCEP extensions for stateful PCE," IETF draft-ietf-pce-stateful-pce-10, Oct. 2014.

[33] J. Vasseur and J. L. Roux (Eds.), "Path Computation Element (PCE) Communication Protocol (PCEP)," IETF RFC 5440, Mar. 2009

[34] R. Casellas, R. Martínez, R. Muñoz, R. Vilalta, L. Liu, T. Tsuritani, and I. Morita, "Control and management of flexi-grid optical networks with an integrated stateful path computation element and OpenFlow controller," IEEE/OSA J. Opt Commun. Netw., vol. 5, no. 10, pp. A57-A65, Oct. 2013.

[35] T. Elhourani, A. Gopalan, and S. Ramasubramanian, "IP fast rerouting for multi-link failures," in IEEE Conference on Computer Communications (INFOCOM 2014), pp. 2148-2156, Toronto, Canada, Apr. 2014.

Lei Liu (M'11) received B.E. and Ph.D. degrees from Beijing University of Posts and Telecommunications (BUPT), Beijing, China, in 2004 and 2009 respectively.

From 2009 to 2012, he was a research engineer at KDDI R\&D Laboratories Inc., Japan, where he was engaged in research \& development on intelligent optical networks and their control and management technologies. He is now with the University of California, Davis, USA. His main research interests include the wavelength switched optical networks, 
elastic optical networks, network control and management techniques (such as MPLS/GMPLS, PCE, OpenFlow), software-defined networking (SDN), network architectures and grid/cloud computing. He has coauthored more than 100 peer-reviewed papers related to the previously mentioned research topics in international journals, conferences and book chapters, and he is the first author of more than 50 of them. He has presented several invited talks, as the first author, in the international conferences, including some prestigious ones such as ECOC'11, ECOC'12 and Globecom'12.

Wei-Ren Peng received the B.S.E.E. degree from National Taiwan University, Taipei, Taiwan, in 2001, and the M.S. and $\mathrm{Ph} . \mathrm{D}$. degrees in electro-optical engineering from National Chiao Tung University, Hsinchu, Taiwan, in 2003 and 2008, respectively.

In 2007, he was a Visiting Researcher at Optical Communications Laboratory, University of Southern California, Los Angeles, under the supervision of Prof. A. Willner, working on optical orthogonal frequency division multiplexing (O-OFDM) experiments. In 2009, he was a Postdoctoral Fellow at National Chiao Tung University, where he was focused on the performance analysis of optical OFDM transmissions. In February 2010, he joined KDDI R\&D Laboratories Inc., Fujimino-shi, Japan, where he has worked as an Associate Research Engineer. He is now with the Futurewei Technologies, USA. He has authored or coauthored more than 100 papers in prestigious international journals and conferences. He also serves as a Paper Reviewer for IEEE and OSA journals. His current interests include digital impairment compensation for single-carrier and multicarrier (OFDM) transmissions.

Ramon Casellas (M'10-SM'12) graduated in telecommunications engineering in 1999 by both the UPCBarcelonaTech university and ENST Telecom Paristech, within an Erasmus/Socrates double degree program. After working as an undergraduate researcher at both France Telecom R\&D and British Telecom Labs, he completed a $\mathrm{Ph} . \mathrm{D}$. degree in 2002. He worked as an associate professor at the ENST (Paris) and joined the CTTC Optical Networking Area in 2006, with a Torres Quevedo research grant.

$\mathrm{He}$ currently is a senior research associate, involved in several international $\mathrm{R} \& \mathrm{D}$ and technology transfer projects. His research interests include network control and management, the GMPLS/PCE architecture and protocols, software defined networking and traffic engineering. He contributes to IETF standardization, and he is a member of the IEEE Communications Society and a member of the Internet Society.

Takehiro Tsuritani was born in Ishikawa, Japan, on November 1971. He received the M.E. and Ph.D. degrees in electronics engineering from Tohoku University, Miyagi, Japan, in 1997 and 2006 respectively.

He joined Kokusai Denshin Denwa (KDD) Company, Ltd. (currently KDDI Corporation), Tokyo, Japan, in 1997, where since 1998, he has been in the Research and Development Laboratories (currently KDDI R\&D Laboratories, Inc.) and has been involved in research on long-haul wavelength- division multiplexing transmission systems and designing and modeling of photonic networks.

Itsuro Morita (M'01) received the B.E., M.E., and Dr.Eng. degrees in electronics engineering from the Tokyo Institute of Technology, Tokyo, Japan, in 1990, 1992, and 2005, respectively.

In 1992, he joined Kokusai Denshin Denwa (KDD) Company, Ltd. (currently KDDI Corporation), Tokyo, where since 1994, he has been with the Research and Development Laboratories. He has been involved in research on longdistance and high-speed optical communication systems, and photonic networks. In 1998, he was on leave at Stanford University, Stanford, CA.

Ricardo Martínez graduated in telecommunications engineering 2002 and received a Ph.D. degree in telecommunications in 2007, both from the Technical University of Catalonia, Spain. Since June 2007 he has been a research associate in the CTTC Optical Networking Area. He has participated in several Spanish, EU-funded (EU FP6, FP7 and CELTIC) and Industrial projects in the context of optical networking. His research interests include GMPLS architecture, distributed control schemes, packet and optical transport networks. He has published over 80 scientific conferences and peer reviewed journal papers.

Raül Muñoz (M'10-SM'12) graduated in telecommunications engineering in 2001 and received a Ph.D. degree in telecommunications in 2005, both from the Universitat Politècnica de Catalunya (UPC), Spain. After working as undergraduate researcher at Telecom Italia Lab (Turin, Italy) in 2000, and as assistant professor at the UPC in 2001, he joined the Centre Tecnològic de Telecomunicacions de Catalunya (CTTC) in 2002.

Currently, he is senior research associate. Since 2000, he has participated in several R\&D projects (FP7 IP STRONGEST and NoE BONE, FP6 IP NOBEL and NOBEL2, FP5 LION, etc.) and technology transfer projects. He leads the Spanish project DORADO. He has published over 50 journal and international conference papers in this field. His research interests include GMPLS architecture, protocols and traffic engineering algorithms for highly dynamic optical transport networks.

Masatoshi Suzuki (S'79-M'84-SM'04-F'06) received the B.E., M.E., and Ph.D. degrees in electronic engineering from Hokkaido University, Hokkaido, Japan, in 1979, 1981, and 1984, respectively.

He joined KDD Research Laboratories (currently KDDI R\&D Laboratories), Tokyo, Japan in 1984. Since 1984, he has been engaged in research on highspeed EA modulators, laser/modulator integrated devices for high-speed optical transmission systems and optical signal processing. In 1995, he proposed and demonstrated the dispersion managed soliton transmission scheme for the first time. He was also involved in the project on the first optically amplified transpacific cable system, TPC-5, and $10 \mathrm{~Gb} / \mathrm{s}$ WDM undersea cable systems which have been applied to many recent undersea cable systems worldwide, such as the Japan-US, PC-1 and TAT-14 
cable systems. His current interest is on IP and photonic networking, including both fixed and mobile networks.

Dr. Suzuki is an Executive Director of KDDI R\&D Laboratories, fellow of IEICE, Fellow of IEEE, Fellow of OSA, and Fellow of KDDI Corporation. He received Best Paper Awards from OEC88, IEICE, and OECC2000 in 1988, 1995, and 2000, respectively. He also received the Achievement Award from IEICE in 2004, the Science and Technology Award from the Minister of MEXT (Ministry of Education, Culture, Sports, Science and Technology) and the Minister Award of advanced technology fromMETI (Ministry of Economy, Trade and Industry) in Japan in 2006. He was an Associate Editor of the IEEE Journal of Lightwave Technologies from 1999 to 2004.

S. J. Ben Yoo (S'82-M'84-SM'97-F'07) received the B.S. degree in electrical engineering (with Distinction), the M.S. degree in electrical engineering, and the Ph.D. degree in electrical engineering with minor in physics, all from Stanford
University, Stanford, CA, USA, in 1984, 1986, and 1991, respectively. He is Professor of Electrical Engineering at University of California at Davis (UC Davis), CA, USA. His research at UC Davis includes future Internet, optical networks, computer interconnects, and advanced photonic integration. Prior to joining UC Davis in 1999, he was a Senior Research Scientist at Bellcore, leading technical efforts in optical networking research and systems integration. He also participated in the advanced technology demonstration network/multiwavelength optical networking (ATD/MONET) systems integration, and a number of standardization activities. He served as an Associate Editor and Guest Editors for a number of IEEE and OSA journals. He is Fellow of the IEEE, OSA, and NIAC, and a recipient of the DARPA Award for Sustained Excellence in 1997, the Bellcore CEO Award in 1998, the Mid-Career Research Faculty Award (UC Davis) in 2004, and the Senior Research Faculty Award (UC Davis) in 2011. 